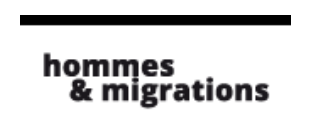

Hommes \& migrations

Revue française de référence sur les dynamiques

migratoires

1286-1287 | 2010

Les migrations subsahariennes

\title{
Mode ethnique. Quand l'Afrique veut en découdre
}

Entretiens réalisés par Térence Carbin et Marie Poinsot

Terence Carbin et Marie Poinsot

\section{Q OpenEdition \\ 1 Journals}

Édition électronique

URL : http://journals.openedition.org/hommesmigrations/1740

DOI : 10.4000/hommesmigrations. 1740

ISSN : 2262-3353

Éditeur

Musée national de l'histoire de l'immigration

Édition imprimée

Date de publication : 1 juillet 2010

Pagination : 148-159

ISSN : 1142-852X

Référence électronique

Terence Carbin et Marie Poinsot, « Mode ethnique. Quand l'Afrique veut en découdre », Hommes \&

migrations [En ligne], 1286-1287 | 2010, mis en ligne le 29 mai 2013, consulté le 21 avril 2019. URL

http://journals.openedition.org/hommesmigrations/1740; DOI : 10.4000/hommesmigrations. 1740

Tous droits réservés 


\section{Mode ethnique Quand I'Afrique veut en découdre}

Entretiens réalisés par Térence Carbin et Marie Poinsot

Être un styliste africain à Paris n'est pas chose aisée. Toujours pas ? Pourtant la mode ethnique, distillée par nombres de grandes marques, a depuis des années investi la moindre parcelle de notre quotidien et présuppose une place largement faite aux créateurs venus d'Afrique. Alors? Hommes et migrations a rencontré quatre jeunes créateurs africains qui travaillent en France. À peine 30 ans, bardés de trophées, désireux de développer leur griffe et d'imposer leur personnalité dans ce monde très sélectif de la haute couture, ils illustrent aujourd'hui le dynamisme et la créativité d'une génération sans complexe qui a choisi de tenter sa chance à Paris. Sakina M'sa, Martial Tapolo, Bill Ruterana et Anggy Haif évoquent leur parcours, leurs visions de la mode et réagissent à cette notion de "mode ethnique" dont les médias affublent trop rapidement les créateurs africains. Une certitude : ils croient au développement de la mode en Afrique et comptent y contribuer.

\section{La mode en mode accéléré}

Contrairement à une idée reçue, venir du continent africain est plutôt un atout pour entrer dans le milieu très sélectif de la mode. Mais il faut avoir un peu de chance, beaucoup de talent et une irrésistible envie de réussir pour franchir les différents obstacles qui permettent de faire rapidement ses classes en Afrique avant de venir en France.

Martial Tapolo, d'origine camerounaise, a interrompu ses études de sociologie et d'histoire de l'art pour s'adonner à sa passion en 2001 : "Très jeune, javais commencé à flirter un peu avec le milieu de la mode en faisant des croquis pour des créateurs que je connaissais. Après trois ans d'université, jai voulu m'épanouir dans une discipline artistique. Je faisais des vêtements à partir de dessins, vendais quelques pièces. Mais n'étant pas couturier, je m'étais limité à des choses simples en lin, des chemises, des robes, des 
sarouels. Le déclencheur? Ma rencontre avec un grand organisateur d'événements de mode à Yaoundé. Tout de suite, il m'a proposé de faire un défilé pour la première élection 'Top model', un gros événement au Cameroun. Le mois d'après, javais une collection de vingt robes à faire. À cette époque j’avais 21 ans, j’étais très flatté. Suite à cette manifestation, mon parcours dans la mode s'est accéléré ; jai été invité dans d'autres pays africains et remporté des prix." 
Anggy Haïf, camerounais également, vit en France depuis 2008. "J'ai choisi la voie de la mode mais en réalité c'est la mode qui m'a choisi. J'ai pu présenter mes créations à la suite d'un spectacle. Puis jai pu développer cette activité de création et remporter des concours locaux. En 2005, jai remporté le concours 'L'Afrique est à la mode' du Fima qui m'a permis de faire un stage de six mois chez Azzedine Alaïa à Paris. C'était parti."

CulturesFrance joue un rôle important dans le repérage de jeunes créateurs en Afrique. Au Niger par exemple, le Festival international de la mode africaine (Fima), organisé par CulturesFrance, repère des créateurs de moins de 35 ans à travers le réseau des alliances ou des centres culturels français. Une première sélection, à Paris, retient 10 candidats de l'Afrique plutôt francophone qui vont préparer leur collection sur un thème choisi pour venir la présenter à Niamey. Ce concours permet d'émerger dans le monde de la mode. C'est un véritable tremplin.

Bill Ruterana, quant à lui, a suivi le même circuit après son fil de bronze du Fima de 2005. Né au Congo mais originaire du Rwanda, il reconnaît que "c'est un réseau spécifique à la politique de la francophonie en Afrique de l'Ouest. Sachant que le Rwanda est aujourd'hui à majorité anglophone, les institutions culturelles françaises peuvent difficilement repérer des artistes rwandais pour les faire participer à des concours de mode. La Grande-Bretagne ne joue pas un rôle équivalent, mais elle est plus ouverte et juge les personnes aux résultats".

Sakina M'sa a un tout autre parcours : "Comorienne, je suis arrivée en France quand javais 7 ans pour rejoindre mes parents. J'ai eu cette chance de pouvoir traverser l'océan et découvrir une autre culture. La mode s'est imposée très tôt comme une évidence. Heureusement, parce que c'est très compliqué. Chaque histoire est particulière et je ne suis pas forcément un exemple. J'ai participé à des concours et reçu des prix comme celui de la Création de la ville de Paris et celui de la Fondation de France en 2002, celui de la Fondation Pinault Printemps Redoute récemment. On voit passer des appels à projet et on tente sa chance. Il n'y a pas de règle et chacun se construit en faisant son chemin avec beaucoup de travail personnel."

\section{Autodidactes et touche-à-tout}

Depuis leur jeune âge, la plupart se lancent dans le stylisme sans passer par une école de couture ou de design. Avant de tenter des concours, ces jeunes ont déjà acquis un savoir-faire dans la couture dans des pays où le sur-mesure est très courant, alors que les vêtements manufacturés sont rares. Débordant d'imagination, avec une furieuse envie de s'éloigner des créateurs traditionnels, ils s'attachent à surprendre en détournant les matériaux naturels au profit de formes délurées. Pour Martial Tapolo, 
"en Afrique, on s'autoproclame styliste dès qu'on a fait des créations, un défilé, etc. Avec le temps, ceux qui veulent émerger se rendent compte que ce n'est pas suffisant et qu'il faut se recentrer sur des corps de métier particulier. J'ai appris en regardant mes piqueurs et mes tailleurs à l'ouvrage. Ce n'est pas en dessinant que l'on devient couturier mais en façonnant. Ma passion, c'est plutôt les matières, les tissus. J'ai cette dextérité qui est un atout manuel et jai su faire sans avoir appris. Je me suis aussi documenté en lisant tous les manuels de coupes et les ouvrages d'art".

Bill Ruterana, à l'origine plasticien, peint beaucoup, inspiré par l'Afrique contemporaine qu'il retrouve dans la

\section{S'installer en France oui, mais pas définitivement. Le métier de styliste nécessite un incessant va-et-vient entre le pays d'origine pour y puiser les inspirations, les couleurs, les matériaux, et la France où l'on crée.} rue, ici en France, comme là-bas : "Je ne suis jamais allé dans une école d'art ; je suis venu à la mode par le dessin, mais au lieu de traiter des thèmes glamour, je parle de la vie, la plus brute, des racines. J'ai été confronté à des gens qui ne nous prenaient pas pour des gamins mais des concurrents. J'ai grandi plus vite que tout le monde et me sens à la hauteur de ceux qui ont fait des écoles de mode. Cette maturité de la vie manque dans le milieu de la mode française qui est si lointain de la réalité des gens."

Anggy Haif a d'abord eu une carrière de danseur et musicien au Cameroun, avant de se consacrer à la couture : "Tout petit, instinctivement, je ramassais des bouts de papier et créais des choses. Je construisais des sacs en carton, etc. Avec le temps, on te dit que tu as du talent et tu t'habilles avec des vêtements que tu crées parce que tu veux être différent. Je ne voulais pas être créateur et vendre, mais je travaillais des matières qui me parlaient. J'ai voulu apporter une nouvelle utilisation de ces matières et un nouveau souffle à l'univers de la mode... Je fais des dessins de vêtements depuis peu, mais jai une bonne pratique de la coupe à plat et de l'assemblage. Je trouve que les jeunes qui sortent des écoles de mode françaises ont de très bonnes bases théoriques mais il leur manque le sens pratique."

Sakina M'sa, "plasticienne du vêtement" depuis 2002, est diplômée de l'Institut supérieur de mode de Marseille, mais dit avoir eu la chance d'être l'élève de Geneviève Sevin-Doering, une éminente costumière de théâtre. "Je l'ai rencontrée à un moment où elle perdait la vue. Elle m'a appris à observer la lumière car, même aveugle, elle pouvait mieux que personne me parler de la lumière. Elle était soucieuse de garder son intégrité, sa liberté et son indépendance. Elle n'était pas du tout dans le milieu de la mode qu'elle n'appréciait guère. C'est elle qui m'a appris mon métier. J'ai ensuite cheminé vers la mode, telle que je voulais la faire. Cela demande du talent, un réseau, le sens du développement commercial. Un peu comme la formule 1." 


\section{Paris, capitale de la mode?}

En Afrique, Paris reste encore un point de repère essentiel pour celui ou celle qui se destine au stylisme. Mais sans ce coup de pouce des réseaux culturels français, il paraît presque impossible de venir en France et de rentrer dans les arcanes de la mode parisienne. Pour Anggy Haif, l'expérience d'un stage à Paris lui a permis de comprendre ce qui relève de cette industrie de la mode. Rentrer dans ce circuit n'est pas toujours à la portée de tout jeune créateur africain, qui se trouve déjà confronté à des problèmes de visas.

Martial Tapolo n'était pas vraiment emballé par l'idée de venir s'installer en France. "J'ai finalement remporté un concours qui m'offrait deux stages dans des maisons de couture en France. J'ai aussi participé à des salons tels que Who's Next, Ethnical Fashion Show, etc. Ce qui me permettait de venir régulièrement en France pour travailler un peu à partir des contacts qui se sont enchaînés. En Afrique, Paris reste encore une destination attractive, car le mythe des grandes marques perdure. On a envie de voir comment cela se passe concrètement. Mais l'enthousiasme redescend tout de suite car on n'est pas accueilli par le boss, on reste cantonné dans un petit box à faire une tâche très limitée. La haute couture est devenue très industrialisée, très administrative et la notion de maître n'existe plus. Et c'est justement ce qu'on attend de notre séjour en France."

Bill Ruterana n'a pas migré pour fuir la guerre, ni pour réussir à Paris. "Je voulais voir les coulisses des grands créateurs. Mais je me suis vite éloigné de Paris. J'ai fréquenté la Biennale internationale du Design de Saint-Étienne qui est très loin du monde parisien et pleine d'énergie. Je voulais voir le vaste monde et partir de Paris. Je ne suis pas contre Paris, mais je peux faire sans Paris. Au final, je souhaite retourner au Rwanda, travailler avec des artisans qui pourraient avoir des productions très fortes artistiquement."

\section{Transnationalisme et transdisciplinarité}

S'installer en France oui, mais pas définitivement. Le métier de styliste nécessite un incessant va-et-vient entre le pays d'origine pour y puiser les inspirations, les couleurs, les matériaux, et la France où l'on crée. Des allers-retours aussi pour mieux mener en Afrique des projets d'ouverture d'ateliers, des espaces de formation ou participer à des regroupements de créateurs africains. Martial Tapolo, malgré le non-professionnalisme, a "pourtant ouvert au Cameroun un grand atelier avec neuf salariés. Les compétences techniques n'y sont pas ; les piqueurs ne maîtrisent pas le patronage, la coupe à plat, le moulage par exemple. Pour réaliser mes envies de haute couture, je devais leur montrer la façon de faire et surtout les encadrer. S'installer à Paris est facilité par la langue commune, les contacts. Mais 
une fois installé, il faut vendre pour faire des collections et c'est plus difficile ici qu'en Afrique".

Bill Ruterana se dit "multicréateur", non fermé sur la mode. Longtemps peintre-décorateur de cinéma en Afrique pour des films africains, américains ou français, il est aussi dessinateur. "Je suis fier d'avoir eu de vraies tentatives de création, même si certaines ont échoué. Ça reste difficile, par exemple, sur le thème des responsabilités de la France dans l'histoire de mon pays. Cest rayé de la liste. Je dois alors m'autoproduire. C'est pour cela que jaime minspirer de la rue, de ce monde très décalé de la mode parisienne. Il faut lever un barrage et cela prend du temps. La musique africaine a commencé il y a très longtemps. La mode va prendre autant de temps pour être reconnue. Des artistes de différentes disciplines devraient s'associer pour provoquer un mouvement plus global."

Pour Anggy Haïf, une carrière doit être internationale. La France n'est pas le seul débouché. D'autres Africains vont dans les capitales de la mode comme New York, Milan,

Londres ou Berlin. Il sait qu'il faut composer avec les avantages des deux localisations : "Les vêtements sont faits ici ou en Afrique, même si le plus souvent je reviens d'Afrique avec des matières pour les travailler en France. On n'est pas vendu en France dans les magasins à grande enseigne car la diffusion est le véritable casse-tête pour nous, les créateurs africains. Il faut avoir des financiers et une capacité de production." Anggy précise: "Les grands groupes financiers comme Pinault et LVMH l'ont compris en soutenant la création en Europe. Mais cet intérêt n'existe pas en Afrique. Les hommes d'affaires africains doivent être sensibilisés en repérant des talents lors des salons de mode. On est obligé d'être polyvalents sans cet appui. Venir en France, c'est se faire repérer et entrer 
dans cette logique industrielle de la mode. Pourtant, en termes d'image, la mode est un vecteur très fort pour changer le regard sur l'Afrique. Même dans les villes africaines, il y a des boutiques de mode partout, mais elles restent très artisanales. Cela ne décolle pas!' L'Internet peut-il développer la visibilité des créateurs? Pas si sûr, car les grands groupes ont déjà capté tous les blogs et cela devient difficile d'être présent sur les sites censés soutenir la jeune création. "Aujourd'hui, il faut $60 \%$ de marketing et $40 \%$ de talent pour réussir. Il faut penser à un concept avant le produit avec toute la stratégie de communication qui supporte le concept. D'ici cinq ans, avec des financements, on pourra trouver de la création africaine dans les grands magasins qui font déjà, dans l'année, 'Le mois de l'Afrique' que je trouve ridicule. On prend encore l'Afrique pour un continent exotique et on la laisse à distance. L'Afrique est aussi moderne." Voilà pourquoi Anggy Haîf a déjà commencé à repérer des jeunes créateurs ici qu'il emmène au Cameroun pour qu'ils transmettent leurs techniques de travail et expliquent la cohérence d'une collection. "Là-bas, la plupart des jeunes n'ont pas reçu de formation dans les métiers de la mode. Il faut qu'ils apprennent qu'à partir d'un thème il faut développer des idées pour rendre crédible leur collection aux yeux de celui qui la regarde. Si tu fais de beaux vêtements sans histoire, c'est comme si tu faisais une robe sans âme. C'est aussi important d'avoir un dossier de presse pour contacter les médias, communiquer. Les jeunes enfin commencent à intégrer ces éléments dans leur structure."

\section{Mode et attaches identitaires}

La couture est d'abord une question de production d'étoffe sur mesure, pour Martial Tapolo. Il y a une sensibilité artistique, une technicité qui entrent en jeu. "En tant que créateur, je suis foncièrement africain et cela est ancré en moi. C'est un univers très coloré, intense, enrichi de souvenirs d'enfance très divers. Un monde qui a bâti ma personnalité et mes sources d'inspiration. Le choix des matières est plus artisanal, plus traditionnel. J'ai beaucoup voyagé dans le pays avec mon père et les danses, les rituels qui restent dans mon esprit sont comme des pierres dans mes créations. Ces souvenirs trottent positivement dans mon esprit. Je me méfie de ces discours très revendicateurs sur la mode ou la création africaines. Moi, je parlerais plutôt de sensibilité où je mêle la modernité qui était mon environnement familial et la tradition qui est une part de mon vécu. La ville occidentale m'inspire aussi."

Sakina M'sa sait bien se situer entre son héritage culturel et une modernité universelle. "Mes ressources personnelles et mes points de repère identitaires sont très importants. Mais, attention, je refuse le credo folklorique sur l'Afrique. Ce n'est pas une forme de déni culturel. Je suis une créatrice de l'univers même si je suis née dans une île 
exceptionnelle. Ce qui m'intéresse est de m'inscrire dans la modernité qui peut être reconnue à New York, à Tokyo, etc. Je ne supporte plus la manière dont on cloisonne les créateurs africains sans leur donner la chance de se développer économiquement. On ne les prend pas au sérieux. Ils restent marginalisés dans un milieu franco-africain restreint. Je n'adhère pas du tout à cet exotisme. Les identités personnelles sont tellement profondes et se nourrissent d'autres identités : je suis comorienne, française, africaine, et fière de vendre au Japon et d'être dans le calendrier international de la couture."

Bill Ruterana s'inspire de l'iconographie de la mode africaine qui comporte plusieurs facettes, mais il conteste une mode occidentale qui cherche à diluer trop tôt et trop vite les ressources culturelles africaines dans la culture européenne.

Or, l'Afrique peut établir une coopération intéressante avec l'Europe en développant des échanges réciproques sur les matières, les formes, les sources d'inspiration. "Etre un créateur rwandais me désigne très bien : petit pays de montagnes, mais immense aussi. J'adapte des situations vécues dans le registre de la mode pour traduire des réalités sociales et historiques. La mode est très contemporaine et très primitive en touchant l'intimité du corps. Elle parle de l'humain. La forme vient de mes mains et le matériel vient de mes yeux, du toucher et de l'odorat. J'essaie de montrer dans mes créations l'Africain qui n'a jamais été montré, comme si je dessinais une nouvelle carte de l'Afrique pour chaque personne, unique, rare, qui exprime une autre Afrique." Pour Anggy Haïf, "la mode s'est engouffrée dans des matières synthétiques qui, aujourd'hui, nous font prendre conscience que l'on est en train de détruire la planète. On retourne aux valeurs naturelles avec la mode bio. En tant que créateur, il faut donc être imaginatif et envisager de nouvelles formes. L'esprit n'a pas de continent. Par exemple, ma nouvelle collection qui s'appelle Black Light célébre la nuit et la lumière. Si un modèle interprète la nuit à Paris, il sera différent d'un autre modèle qui évoque la nuit en Afrique : les matières vont changer avec des imprimés ou des écorces qui auront une coloration plus ethnique. Les créateurs sont influencés par leurs voyages, leurs rencontres, leurs expériences de la rue. En Afrique, on n'a pas de tendances ou de 'fashion weeks' comme en Europe. Ces tendances sont décidées par les créateurs, mais c'est aussi un mélange de l'air du temps traduit par un univers propre."

\section{Quid de la "mode ethnique" ?}

Anggy Haïf n'a jamais aimé ce terme qui apparaît dans les années quatre-vingt-dix, à la faveur du succès de la génération des créateurs comme Alphadi ou Xuly Bët. "C'est une problématique qui tend à ghettoïser les créateurs africains et à nier le mode d'inspiration de la mode ou les influences. Par exemple, quand Hermès travaille sur l'Inde, on ne dit pas que c'est de la mode ethnique car cela reste du Hermès. Moi, je ne dis pas que 
je fais de la mode africaine mais de la mode qui s'inspire de mon univers qui est africain. Ce ghetto limite l'entrée du créateur africain pour entrer sur le marché en prétextant souvent que la clientèle n'est pas africaine, à quoi je réponds que ma clientèle est à $90 \%$ européenne. Ce n'est pas une mode destinée aux Africains. C'est aussi en partie de la faute de nos aînés qui n'ont pas su apporter sur le marché de la mode quelque chose de frais et de contemporain. Leur mode est restée basique dans les formes et les matières et ils n'ont pas apporté un nouveau souffle à leurs créations qui sont restées très en arrière. Nous sommes une génération née en ville et nous ne nous limitons pas à faire des cache-sexe ou des jupes en paille. Il faudrait moderniser nos racines culturelles." Il ajoute : "J'ai aussi ma ligne de cœur qui est celle de la création - la ligne ancestrale et du retour aux sources - qui est là pour montrer mon univers, mes origines, les capacités créatives de la culture africaine que je réalise avec des matières naturelles qui ont été oubliées. Je me suis mis à les travailler pour montrer à la jeunesse qu'il ne faut pas avancer sans connaître son passé. Et je vois que, depuis dix ans, tout le monde fait son retour aux sources et aux fibres naturelles, si bien qu'aujourd'hui, la mode africaine n'existe plus. Tous les grands créateurs utilisent ces matières, mieux exploitées parce qu'ils ont les budgets pour les traiter. Nous, les Africains, on est un peu devancé et on a l'impression qu'il n'y a plus rien qui appartient à l'Afrique. Chez Dior ou chez Fendi, vous retrouverez les mêmes matières."

Sakina M'sa, elle aussi, réagit vivement quand on lui parle de "mode ethnique". Elle fait d'emblée une différence entre identité et exotisme. "Ce qui me dérange, c'est l'exotisme du 'y-a-bon-Banania' qui s'exprime, ce sont les années trente, mais aujourd'hui. Il faut être prudent et respectueux des individus et de leurs cultures. Mon identité c'est la sensibilité et l'émotion avec lesquelles je compose mon défilé et que l'on lperçoit aussi dans mon vêtement. On peut proposer une ligne de vêtement qui soit influencée à la fois par Schiaparelli, Vionnet, Balenciaga, et aussi des imprimés que l'on retrouve dans mon pays, sans pour autant en faire un étendard. J'espère que ma mode me ressemble. La haute couture ne me regarde pas avec cet exotisme. La haute couture, c'est une quinzaine de maisons avec des marques déposées. Le président de la fédération de la couture, Didier Grumbach, est très ouvert sur l'extérieur et fait en sorte que des jeunes puissent s'exprimer. Il a inventé ce concept d'inviter chaque année des jeunes créateurs à la chambre syndicale en toute modestie. On est loin de l'atelier de Balenciaga avec 40 personnes. Nous on est trois avec un projet d'insertion sociale, cela fait toute la différence. Les jeunes créateurs africains doivent sortir de cet exotisme pour rentrer dans cette économie de la mode où 'l'ethnique' peut être sur une saison. Marc Jacob peut travailler le tissu batik sans être ethnique."

Bill Ruterana rejette de manière plus véhémente cette appellation ethnique. "Je ne veux pas qu'on m'appelle 'ethnique' mais qu'on me juge 'ethnique' car je viens d'ailleurs. Mais je ne suis pas la carte postale aseptisée et consensuelle de l'Afrique, mais l'Afrique blessée. Comme créateur ici, je dérange plutôt en produisant du flou. Les journalistes spécialisés dans 
la mode ne nous interrogent pas sur l'immigration et vice versa. Je suis une image-choc pour ces milieux qui n'arrivent pas à me capter. Je suis l'immigration et la mode à la fois et je souhaite que l'on en parle."

La création africaine sortira-t-elle de cette ethnicisation? Martial Tapolo nous donne un début de réponse: "Je ne compte pas m'éterniser en France et j’espère que la mode africaine s'affirmera en Europe. Des recherches sont faites au Kenya, par exemple, par les grandes marques qui s'inspirent beaucoup du tribal ou de l'ethnique. La haute couture parle d'apparat très présent en Afrique, de superposition, de grandiose. Les échanges donnent des chances à d'autres cultures de s'exprimer. L'Europe s'essouffle et commence à regarder autrement les autres cultures. Depuis des décennies, on parle de mode tribale avec des pièces très impressionnantes dans les défilés de haute couture. Des couturiers comme Yves-SaintLaurent ont joué un rôle précurseur pour métisser la mode. L'utilisation des influences ethniques ne se réduit pas seulement à l'Afrique, l'Asie est présente aussi depuis longtemps."

\section{La mode, une économie sociale et solidaire}

Dans ce monde sélectif de la mode, Sakina M'sa a su combiner, depuis 1998, démarche professionnelle et ateliers de couture dans les quartiers, afin de relancer l'insertion des populations fragilisées. C'est une combinaison fondamentale qui lui permet de garder son intégrité. "Je viens d'un milieu plutôt ouvrier. Un jour, en accompagnant ma mère au centre social de mon quartier, j'ai eu l'impression qu'on lui parlait 'petit nègre'. Elle était très gênée que sa fille assiste à ça. Je me suis jurée que, quelle que soit l'activité que je ferai plus tard, je garderais le contact avec ma communauté d'origine." En effet, après son diplôme, elle arrive en Seine-Saint-Denis, se rend dans le centre social du quartier où elle réside et propose des cours de couture "à défaut d'avoir un stage dans une grande maison de couture" pour partager ses connaissances acquises à l'école de mode avec un public d'adolescents. "J'ai ensuite donné des cours à des femmes, puis à des personnes âgées dans une maison de retraite. C'était une collaboration très enrichissante de travailler avec des personnes sereines qui ne se plaignent pas alors qu'elles vont peut-être mourir tout à l'heure; cela redonne une signification à la plainte, à la revendication. J'ai continué ces activités qui correspondent à des engagements personnels depuis cette promesse faite à ma mère quand jétais gamine. Quand jai monté ma société et organisé des défilés, cette communauté sociale me suivait, que ce soit dans un centre de quartier ou un endroit prestigieux. Je faisais cette activité d'insertion sans m'en rendre compte, comme un prolongement de moi-même. C'est un audit de l'Essec qui m'a dit un jour que j'étais ce qu'on appelle un 'porteur de projet dans l'économie sociale et solidaire.' On a alors formalisé ce qui était en cours." 
Sa méthode s'appuie sur la décomposition puis la recomposition du vêtement. Elle est célèbre pour sa technique du "tissu enterré" ou "planté" qui subit un vieillissement naturel par oxydation lente. "J'ai toujours eu envie d'établir la traçabilité du vêtement pour montrer toutes les étapes dans la production d'une collection. Il y a aussi ce refus de ce qu'on peut appeler la 'société kleenex', on prend, on jette, etc. Je fais toujours attention à la façon dont je consomme et je trie. Le recyclage est une manière de se dire qu'il $y$ a de trop, qu'il faut regarder et faire avec ce que l'on a déjà. C'est très important de dire à la jeunesse qu'elle peut créer, même si elle a l'impression parfois d'être démunie."

Car Sakina ne veut surtout pas tricher avec les gens, quitte à ne pas leur faire plaisir. Femme d'exigence, quand le dialogue ne passe pas, elle sait prendre sa place et surtout faire passer ce qui lui importe. "L'atelier de la désobéissance" au Petit Palais reste pour elle une expérience formidable. "Je suis une militante et jai beaucoup travaillé avec le DAL, AC. Je regarde ces personnes avec une certaine objectivité, sans misérabilisme. Finalement, dans ce lieu prestigieux qu'est le Petit Palais, on a d'abord regardé les tableaux de la collection en les interrogeant, puis on les a interprétés en confectionnant ensemble une collection de haute couture. Je me suis dit que le plus important était l'exposition elle-même parce que l'on a été exigeant dans la mise en espace des modèles. Les amateurs d'art ont été bluffés, même si le travail restait artisanal." Une autre résidence, au 104, vient de s'achever en juillet 2010. Elle a regroupé "des publics du quartier de la Goutte-d'Or que l'on a fait venir, des publics du 104 et des publics 'Télérama'. C'est très important d'ouvrir les projets artistiques à des publics très différents".

\section{Mode et développement solidaire : demi-mesures}

Anggy Haïf tire de son expérience en France un sentiment mitigé : "Paris reste très codifié et très fermé sur les cursus des écoles françaises. On te juge en fonction de tes références et non de ton talent ou de ton travail. Après, il faut beaucoup travailler pour rentrer dans les réseaux et avoir une reconnaissance." Au Cameroun, avec des exemples de réussite comme le sien, les jeunes ont compris que l'on peut partir de rien et bâtir une collection avec des matières naturelles et en utilisant l'environnement. Il y a un potentiel sur place à exploiter. Mais il reconnaît que "les mentalités ne sont pas très ouvertes. Il faut convaincre les jeunes de se rassembler pour se lancer sur un chemin qui va aboutir. Eux, ils ne voient pas forcément les choses de cet æil-là. Comme je suis un jeune créateur qui vit à Paris de son travail, je suis plus crédible et ils commencent à me suivre. Les générations qui nous ont précédés travaillaient sans se préoccuper de l'avenir. L'association de créateurs permet de pouvoir faire évoluer la mode et d'avoir un dynamisme plus important que les â̂nés." 
Martial Tapolo est membre de plusieurs associations en Afrique associées à des corps de métier, pour soutenir la manufacture de tissu et obtenir que certaines matières produites localement, comme le coton, soient enfin traitées. "Certains tissus traditionnels produits à la main par des vieilles femmes vont cesser d'être fabriqués par manque de relève. Lusage de certaines matières rares, comme le raphia, les bois tropicaux, les cornes n'a pas dépassé le stade d'un usage très rituel qui ne les rend pas aptes à la fabrication. Il faut sonner l'alarme et se battre pour faire en sorte que ces tissus ne disparaissent pas. Il n'y a pas assez de moyens en Afrique pour mener cette guerre-là. Les politiques doivent simpliquer pour obliger les hommes d'affaires à faire des investissements et soutenir le développement des filières de la confection."

Se draper de la cape de styliste en Afrique revêt une autre dimension quand on franchit les frontières de la mode en France. L'énergie déployée sur le continent s'effiloche en se confrontant à la réalité de la mode parisienne qui garde à distance la création africaine avec l'étiquette "ethnique", alors qu'elle s'inspire des traditions de ces pays et y pille les ressources naturelles. Les stylistes africains voient tout de même se développer des pratiques artistiques et culturelles dans le domaine de la mode, notamment en Afrique de l'Ouest, ce qui redonne de la valeur à la création.

Même si cela reste encore embryonnaire, il y a encore un gros manque d'investissements en matière de production en propre de richesses, alors que la demande existe. L'offre n'est pas encore disponible sur place par manque d'outil industriel et la clientèle doit aller en Europe pour acheter des produits d'inspiration africaine. Malgré tout, les créateurs gardent un optimisme certain quant au développement des coopérations plus équitables entre l'Afrique et l'Europe et, sans attendre, participent eux-mêmes au développement de leur pays sans s'enfermer dans une mode ethnique. Le monde est l'horizon qui trame leur mode.

Sites Internet 